\title{
Correction to: Ferromagnetic Properties of Five-Period InGaMnAs/GaAs Quantum Well Structure
}

\author{
YOUNG H. KWON, ${ }^{1}$ SEJOON LEE, ${ }^{2}$ WOOCHUL YANG ${ }^{2}$ \\ CHANG-SOO PARK, ${ }^{3}$ and IM TAEK YOON (1) ${ }^{1,4}$ \\ 1.-Quantum Functional Semiconductor Research Center, Dongguk University, Seoul 100-715, \\ Korea. 2.-Department of Physics and Semiconductor Science, Dongguk University, \\ Seoul 100-715, Korea. 3.-Department of Physics and Quantum-Function Research Laboratory, \\ Hanyang University, Seoul 133-791, Korea. 4.—e-mail: ityoon@dongguk.edu
}

\section{Correction to:}

JOURNAL OF ELECTRONIC MATERIALS, Vol. 46, No. 7, 2017

https://doi.org/10.1007/s11664-016-5036-x

In the original article some funding information in the Acknowledgements section was inadvertently omitted. Following is the corrected text.

This research was supported by the Basic Science Research Program through the National Research
Foundation of Korea (NRF) grant funded by the Ministry of Education, Science and Technology (MEST) (NRF-2016R1D1A1B03930992), (NRF-2016 R1A6A1A03012877), and (NRF-2014M2B2A4032 178), as well as under the framework of the international cooperation program managed by the National Research Foundation of Korea (2015K2A1B8068543). 